\title{
A Case of Isolated Unilateral Glossopharyngeal Nerve Palsy
}

\author{
Monisha K. Savarimuthu, MBBS, DPM and Anil K. Nair, MD
}

\begin{abstract}
Isolated palsy of the glossopharyngeal nerve is rare. We report the case of an elderly patient with unilateral right glossopharyngeal nerve palsy secondary to extra cranial ischemia. On examination there was no other deficit other than an absent right gag reflex. She was diagnosed clinically with ischemic stroke of the ninth nerve, and her daily dose of aspirin was increased from $81 \mathrm{mg}$ to $325 \mathrm{mg}$. Magnetic resonance imaging of the brain showed a normal brainstem and cerebellum with patent intracranial circulation. Total resolution of the paralysis was seen 2 months later.The possible mechanisms suspected were diabetic or hypertensive stenosis of the vasa nervorum or compression of the ninth nerve by an internal carotid artery dissection or aneurysm. This article discusses the various etiologies and mechanisms of this rare condition. It is unique because of the nerve's location and relationship to other structures.
\end{abstract}

Keywords: Dysarthria; Gag reflex; Glossopharyngeal nerve; Internal carotid artery; Superior constrictor muscle; Stylopharyngeus muscle

$\mathrm{I}$ solated glossopharyngeal nerve palsies (IGNP) are almost unheard of, which is due to the proximity of the glossopharyngeal nerve to other structures such as the eighth, tenth, and eleventh cranial nerves (Figures 1 and 2). ${ }^{1-3}$ IGNP can occur from damage to the medulla at its exit, across the cerebellopontine angle and the flocculus, its sensory ganglion at the jugular foramen, as it crosses the internal carotid artery anteriorly in the neck, at the styloid process located posterolaterally, or at the pharyngeal plexus in the posterior pharyngeal wall (PPW). Unilateral peripheral motor neuron causes are grouped into medullary, intracranial, and extra cranial. Vascular pathology at the medulla is more common than posterior fossa neoplasia and syringomyelia. Intracranial lesions from the medulla to the jugular foramen include inflammation such as meningitis, jugular foramen neuromas, glomus jugular tumor, sigmoid venous thrombosis, and basilar skull fractures. Ischemia, infection, compressions from retropharyngeal spaces, and internal carotid artery dissection are a few plausible extra cranial causes. Myoneural junction lesions are mainly bilateral and, thus, not an etiology for IGNP.

\section{Case History}

A woman, aged 71 years, with hypertension, type 2 diabetes mellitus, hyperlipidemia, and who was a former tobacco smoker was referred to us for speech difficulties. The difference in her speech was first noticed by an orthopedist, a month prior when she went for a follow-up appointment. Apart from mild dysphagia to liquids, there was no history of nasal regurgitation, taste changes, or other cranial nerve and neurological deficits. Other minor medical history included occasional dizziness and reduced hearing that were attributed to vertigo and senile hearing impairment. On examination, she was afebrile, normotensive, and dysarthric. She scored a 1 on the NIH stroke scale. It was difficult to assess her speech changes, as we did not know her baseline. She could not
Corresponding Author: Monisha K. Savarimuthu, MBBS, DPM, Christian Medical College and Hospital Vellore, Department of Psychiatry, CMC College Road. Near Police Station, Bagayam, Vellore, Tamil Nadu 632002 INDIA, Email: docmos22@gmail. com
Received: July 4, 2018

Ist Revision: April 5, 2019

2nd Revision: May 18, 2019

Accepted: June 14, 2019 


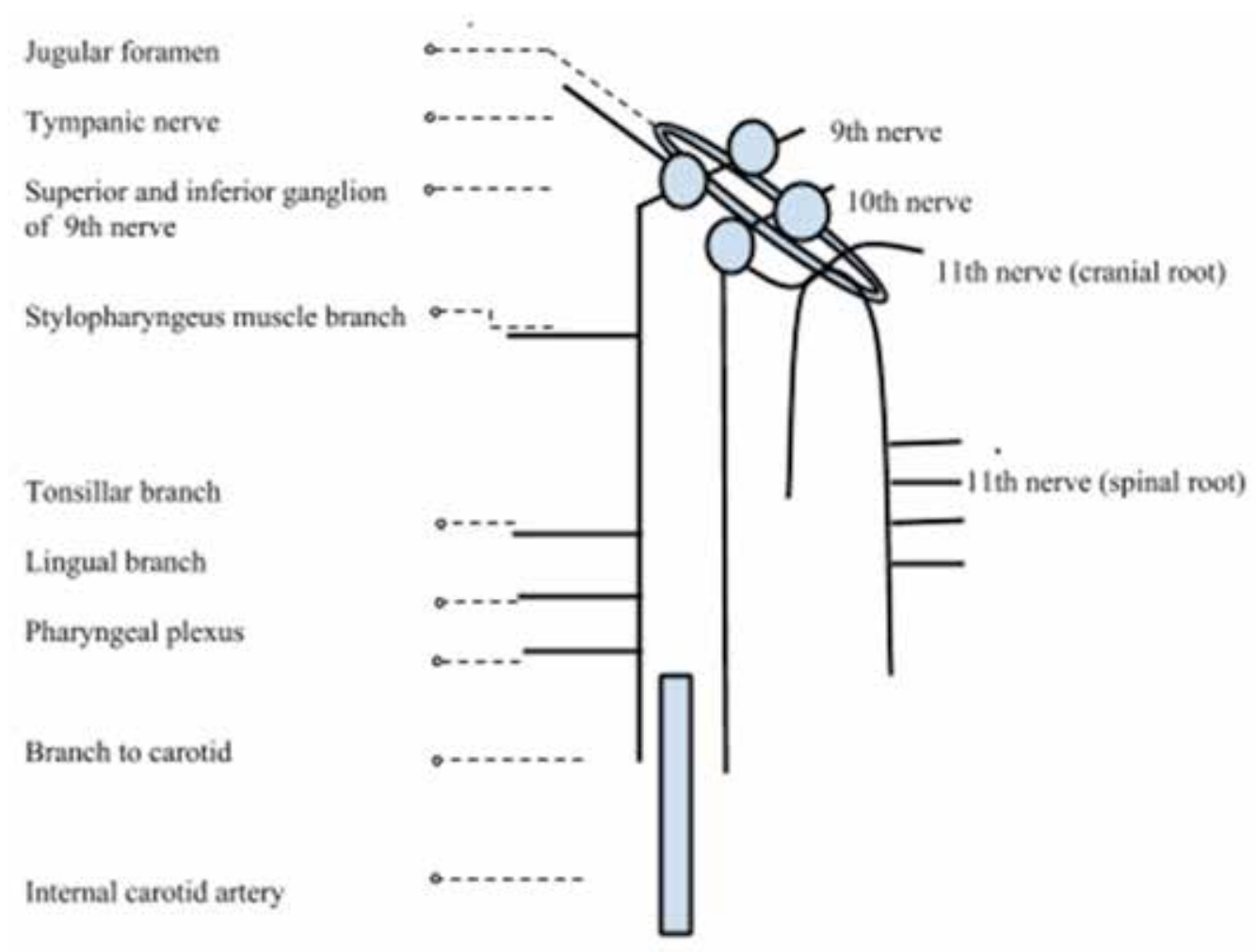

Figure 1. Schematic drawing depicting the extra cranial ninth cranial nerve with its branches and posteromedial relations. ${ }^{1,2}$

repeat the words "Thanks" and "Huckleberry" clearly, but she was able to repeat "Mama", "Tip-top", "Fifty-fifty", and "Baseball player". There was absence of bilateral contraction of the PPW after a right PPW stimulus. She had left-sided elevation of the PPW on touching the left PPW. Movement of the tongue and facial muscles were intact. With consent, a picture and a video of the gag reflex were obtained (though not presented here because of poor quality). The rest of her cranial examination and neurological examination were unremarkable except for a positive Dix Hallpike maneuver of the left.

Magnetic resonance imaging of the brain was performed and showed nonspecific findings of a tiny old lacunar infarct of the left thalamus, left inferior basal ganglia perivascular dilation with periventricular, and subcortical white matter hyper intensities. No clear medullary involvement was seen. Magnetic resonance angiography was non-specific. Glycemic indices were high with a hemoglobin A1c of $11.2 \%$.

The patient was diagnosed clinically with ischemic stroke of the ninth nerve. Differentials considered are discussed in detail below. Her daily dose of aspirin was increased from $81 \mathrm{mg}$ to $325 \mathrm{mg}$. Total resolution of the paralysis was seen 2 months later after sugars were corrected.

\section{Discussion}

The glossopharyngeal nerve is mainly a sensory, though mixed, cranial nerve. The motor root of the ninth nerve, which supplies both the stylopharyngeus and the lateral portion of the superior constrictor muscle, takes origin from the nucleus ambiguus of the medulla; the parasympathetic fibers to the parotid gland travel via the inferior salivatory nucleus and the taste fibers from the posterior third of the tongue synapse in the nucleus of tractus solitarius. ${ }^{1}$ The superior and petrosal ganglion of the ninth nerve receive the sensory fibers that synapse in the thalamus and cortex via the spinal nucleus of the trigeminal nerve. ${ }^{1}$ They innervate the tonsils and its fauces, posterior pharynx, soft palate, posterior third of the tongue, carotid glomus and sinus, pinna and external auditory canal. ${ }^{1}$ Supranuclear innervations from the precentral gyrus to the above nuclei are partly crossed and partly uncrossed. The ninth nerve and its muscles, the stylopharyngeus and lateral portion of the superior constrictor muscle, are supplied by the branches of the ascending pharyngeal artery of the external carotid system. ${ }^{4}$ The ninth nerve is also supplied by the vertebrobasilar system. ${ }^{5}$

The anatomy of the pharyngeal wall and the innervation are unclear, and several studies have revealed different innervation patterns in these muscles. ${ }^{6-8}$ Even the physiology of the gag reflex is ambiguous. Few reports account for an afferent glossopharyngeal that synapses in the ipsilateral nucleus ambiguus and sends out its efferent via the vagus. ${ }^{9}$ Others describe an intervening spinal nucleus and trigeminothalamic tract that receives the afferent impulses of the ninth and sends it to bilateral nucleus ambiguus, resulting in bilateral contraction of the PPW. ${ }^{1,10}$ The motor response is generally unclear and includes pharyngeal contraction with or without contraction of the medial aspect of the posterior pharyngeal wall, elevation of the soft palate, tearing, withdrawal of the head, coughing, and retching. ${ }^{11}$ The visible portion of the gag 


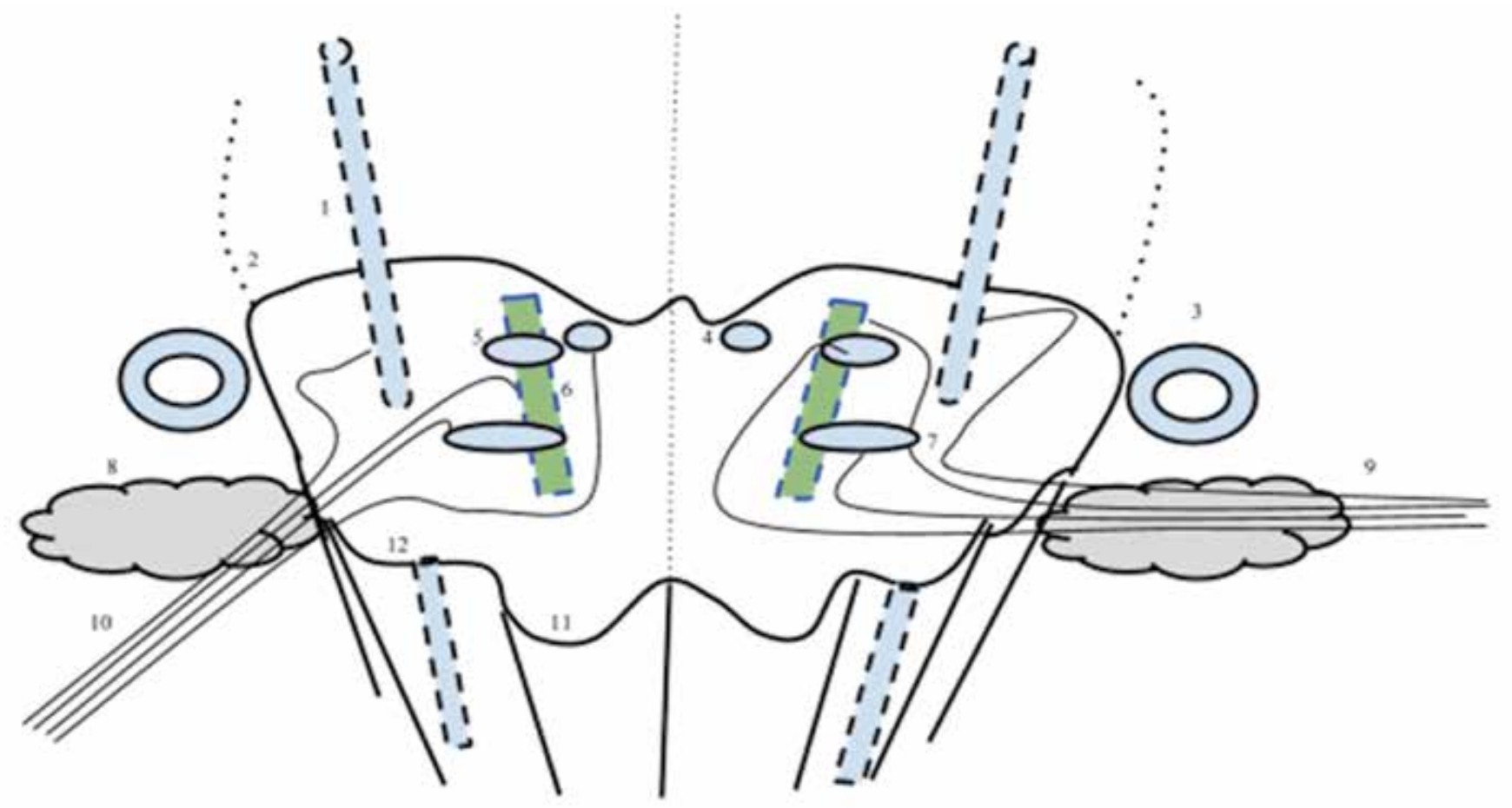

Figure 2. Schematic drawing showing a cross section of the medulla illustrating the nuclei and the intracranial relations of the ninth nerve.1,3 (1) Spinal nucleus of the trigeminal; (2) Ponto medullary junction; (3) Eighth cranial nerve with cerebellopontine relation to the ninth nerve; (4) Dorsal motor nucleus; (5) Inferior salivatory nucleus; (6) Nucleus tractus solitarius-upper third to the ninth nerve and middle third to the vagus; (7) Nucleus ambiguous; (8) Flocculus of the cerebellum; (9) Glossopharyngeal rootlets; (10) Vagus nerve; (11) Pyramid; (12) Inferior Olivary nucleus.

reflex is the medial portion of the superior constrictor muscle (SCM), innervated by the pharyngeal branch of vagus (X-ph).

A unilateral absence of gag reflex with an intact uvula, symmetrical palatal elevation, midline tongue, and lack of hyper-nasal speech and hoarseness of voice, signifies a lower motor neuron ninth nerve palsy, mainly ninth sensory defect. We suspected an extra-cranial affect as medullary involvement would typically include multiple nerves, which are the taste fibers of the tractus solitaries and the ninth through twelfth cranial nerve palsy. (The ninth through eleventh cranial nerves arise from the nucleus ambiguus; the nucleus tractus solitarius and the twelfth nerve motor nucleus also lie in the medulla). The absence of other cranial nerve involvement and a negative magnetic resonance brain imaging points towards an extra cranial lesion of either nerve or muscle. We suspected an ischemic basis with differentials being diabetes or hypertension predominantly affecting the vasa nervorum or Schwann cell.

Cranial neuropathies are extremely rare $(0.05 \%) .{ }^{12}$ However, individuals with diabetes have a $29 \%$ higher risk of being affected than do those without diabetes with regards to Bell's palsy. ${ }^{13}$ Age over 50 years, long-term illness and poorly controlled diabetes (as in our patient), and diabetes itself are risk factors for cranial neuropathy. However, early occurrence in diabetes has been reported with poorly controlled blood sugars. ${ }^{13}$ Other clues to diabetic neuropathy are co-existing retinopathy or nephropathy, which were not present in our patient. Most diabetic cranial nerve palsies involve nerves III,
IV, VI and VII, with spontaneous resolution in 2 to 3 months. ${ }^{14}$ Neurovascular damage from stenosis (local embolization or diabetic vasculopathy of the vasa nervorum) and hyperglycemic neuronal damage are the proposed mechanisms in diabetic cranial neuropathy. Aldo-keto reductase family 1 member $B$ (AKR1B1) polymorphisms might also influence the decline of nerve function. Diabetic vasculopathy consists of thickening of the basement membrane and destruction of the blood nerve barrier from inflammation. This impairs tissue perfusion, causes red blood cell seepage and ensuing local edema with endoneurial and perineurial vasculitis. ${ }^{15}$ Local embolization from local inflammation results in focal nerve hypoxia causing either segmental demyelination or wallerian degeneration. ${ }^{16}$ However, local or distal embolization of one arteriole would not explain ischemia of the nerve, as there is generally good collateral circulation available within the nerve. Vasculopathy could also be from hypertension induced basement membrane hyalinization. ${ }^{16}$ Neuronal damage from hyperglycemia could be from the polyol flux pathway, as it explains the reversibility of symptoms despite long standing diabetes, unknown glycemic status, and lack of any metabolic control intervention by us. However, hypoxic ischemia of damaged vessels and capillaries is favored over polyol flux theory for cranial nerve palsies. ${ }^{17}$ Schwann cell damage without axonal injury can occur. This is the most likely structure to be affected, as it is the closest structure to the blood nerve barrier and is most sensitive to hypoxia and toxic metabolites; injury can be reversed within 3 months, if no advanced glycation end product molecules have set in. ${ }^{16,18}$ 
Compression of the ninth nerve alone from internal carotid artery dissection and aneurysm has been reported. Hematoma at the pre-petrosal (C2 level) level of the internal carotid artery affects only the ninth nerve, sparing the other cranial nerves, with $50 \%$ of patients presenting with Horner's syndrome. ${ }^{19}$ Extracranial internal carotid artery aneurysm affecting only the ninth nerve has been reported. ${ }^{20}$ Hematomas or aneurysms could either compress or stretch the nerve, causing segmental demyelination, which is reversible in 3 months. ${ }^{18}$ Segmental demyelination occurs by intussusception of the internodes at the site of pressure into the adjacent myelin sheath. ${ }^{21}$ Interruption of the nutrient vessels supplying the nerve is also suspected, but to a lesser extent, due to dual innervation of the ninth nerve at the jugular foramen and prepetrosal area and rich collaterals within the nerve (dual innervation from the vertebrobasilar system and the external carotid system comprising of the ascending pharyngeal or occipital or middle meningeal arteries). ${ }^{5}$

Lyme Disease infection is also possible given an endemic area, but the resolution of symptoms without the need of antibiotics, the absence of a classic history, and the infrequent involvement of lower cranial nerves makes it less likely. ${ }^{22}$ Lyme Disease testing was negative as well in our patient. The possibility of a pure dysarthria was not considered at this point because of the absence of a hyperactive gag reflex and an insignificant brain imaging. Ischemic stylopharyngeus does not have clinical relevance, as it functions as a pharyngeal elevator in conjunction with the other pharyngeal muscles and cannot be separately identified or tested. The lesion can also be idiopathic.

Based on the theory of bilateral motor response of the gag reflex, absence of right PPW contraction on applying left pharyngeal stimulus could also mean unilateral paralysis of the right SCM from ischemia to the right X-ph. However, we disagree with this possibility in our patient for the following reasons. Based on a fetal study, SCM and palatopharyngeus (PP) are connected in fetal life; hence, their nerve should be common (based on Shimokawa's concept). ${ }^{7,8}$ Thus, ischemia to the X-ph branch, being a common nerve, would also affect the PP presenting as hyper nasality, soft palate paresis, and nasal regurgitation unilaterally, which were all absent in our patient. Arterial ischemia of SCM is unlikely due to rich anastomosis between the arteries of this region. Pure ninth (motor) nerve lesion would not affect the motor response, as it innervates the lateral hidden portion of the SCM.

Lastly, the most interesting point to note is the role of an orthopedic surgeon in the diagnosis. This raises the question of the frequency of the occurrence of dysarthria seen in the field of orthopedics. There have been occurrences, and this is thus a potential topic to explore. ${ }^{23,24}$ Our orthopedist was astute enough to pick up on the change in voice to trigger a referral.
Other points to highlight would be the following:

- An absent unilateral gag reflex suggests an abnormality, unlike an absent bilateral gag reflex which is seen in healthy individuals. ${ }^{25}$

- Hypertension from baroreceptor reflex damage would be a feature of bilateral ninth nerve lesions rather than unilateral.

- Compressions such as internal carotid artery dissections or aneurysms must be considered and ruled out as the evidence is easily obtainable.

- In $8 \%$ of the population, ninth nerve palsy can present with nasal regurgitation due to common motor nerve supply to the levator veli palatini (LVP) and SCM. In such instances, LVP does not receive X-ph nerve innervation. ${ }^{8}$

- Aspirin, insulin, and alpha lipoic acid (ALA) have been tried in the treatment of diabetic cranial nerve palsies. ${ }^{26-28}$ Near complete resolution was seen within 3 weeks of intensive insulin therapy in type 2 diabetics. Significant improvement occurred within 10 days of ALA therapy. Aspirin was ineffective in prevention. The best preventive therapy is optimum glycemic control.

- Intensive insulin therapy speeds recovery compared to conventional insulin therapy by 5 months. ${ }^{13,26}$ Conventional insulin therapy is equivalent to spontaneous remission based on duration of resolution (3 to 6 months). ${ }^{13,14}$

- Medical therapy with antiplatelet agents was sufficient in treating carotid dissection cranial palsy. ${ }^{29}$

- Glucocorticoids help preserve the blood nerve barrier by up-regulating the expression of junctional proteinclaudin. ${ }^{30}$

\section{Conclusion}

Differentials of diabetic or hypertensive vasculopathic changes should be considered in the evaluation of isolated glossopharyngeal nerve palsy in the absence of other suggesting lesions. Other differentials to consider are compressive neuropathy (nerve or Schwann cell) or compressive ischemia, Lyme Disease-related neuritis, and idiopathic. Recovery can be accelerated by 2 months with supportive therapy with nerve nutrients and intensive insulin. Despite several recent studies on pharyngeal muscles innervation, the anatomy is unclear. The following main points remain: unilateral absent gag reflex is abnormal and mainly a ninth sensory lesion. Rarely it can be accompanied with nasal regurgitation (LVP paresis). Palatal paresis, hyper-nasality, and nasal regurgitation is mainly a feature of tenth pharyngeal nerve lesion. A proper understanding of the gag reflex in humans is essential.

\section{Acknowledgements}

The authors thank Charles A. Dicecca, MD for assisting in the preparation of this article.

\section{References}

1. Gray H. The Glossopharyngeal Nerve. In: Gray's Anatomy: Anatomy of the Human Body [online]. Available at http:// www.bartleby.com/107/204.html. Accessed June 13, 2014. 
2. Accessory nerve (XI): Schema. In. Netter FH. Atlas of Human Anatomy. 5th ed. Philadelphia, PA: Saunders; 2011:126.

3. Wilson-Pauwels L, Stewart PA, Akesson EJ, Spacey SD. Cranial Nerves: Function \& Dysfunction. 3rd ed. Shelton, CT: People's Medical Publishing House-USA; 2010.

4. Standring S, ed. Gray's Anatomy. The Anatomical Basis of Clinical Practice-40th edition. Spain: Churchill Livingstone Elsevier; 2008

5. Hendrix P, Griessenauer CJ, Foreman P, et al. Arterial supply of the lower cranial nerves: A comprehensive review. Clin Anat. 2014;27(1):108-117.

6. Sakamoto Y. Classification of pharyngeal muscles based on innervations from glossopharyngeal and vagus nerves in human. Surg Radiol Anat. 2009;31(10):755-761.

7. Abe S, Fukuda M, Yamane S, et al. Fetal anatomy of the upper pharyngeal muscles with special reference to the nerve supply: is it an enteric plexus or simply an intramuscular nerve? Anat Cell Biol. 2013;46(2):141-148.

8. Shimokawa T, Yi SQ, Izumi A, et al. An anatomical study of the levator veli palatini and superior constrictor with special reference to their nerve supply. Surg Radiol Anat. 2004;26(2):100-105.

9. Jones HR Jr, Srinivasan J, Allam GJ, Baker RA. Netter's Neurology-2nd edition. Philadelphia: Elsevier Saunders; 2012

10. Neuroscience Resource Page. Brainstem Nucleus Ambiguus. Available at http://www.neuroanatomy.wisc.edu/Bs97/TEXT/ P9/intro.htm. Accessed September 5, 2019.

11. Hughes TA, Wiles CM. Palatal and pharyngeal reflexes in health and in motor neuron disease. J Neurol Neurosurg Psychiatry. 1996;61(1):96-98.

12. Boulton AJM, Vinik AI, Arezzo JC, et al; American Diabetes Association. Diabetic neuropathies: a statement by the American Diabetes Association. Diabetes Care. 2005;28(4):956-962.

13. Semiz S, Fişenk F, Akçurin S, Bircan I. Temporary multiple cranial nerve palsies in a patient with type 1 diabetes mellitus. Diabetes Metab. 2002;28(5):413-416.

14. Bedlack R. The Management of Diabetic Neuropathy and Glycemic Control in Long Term Care Facilities (Part II of III). Available at http://www.annalsoflongtermcare.com/ content/part-ii. Accessed February 23,2015.

15. Said G, Lacroix C, Lozeron P, Ropert A, Planté V, Adams D. Inflammatory vasculopathy in multifocal diabetic neuropathy. Brain. 2003;126(2):376-385.

16. Eames RA, Lange LS. Clinical and pathological study of ischaemic neuropathy. J Neurol Neurosurg Psychiatry. 1967;30(3):215-226.

17. Said G. Focal and multifocal diabetic neuropathies. Arq Neuropsiquiatr. 2007;65(4B):1272 1278.

18. Quan D, Bird SJ. Nerve Conduction Studies and Electromyography in the Evaluation of Peripheral Nerve Injuries. The University of Pennsylvania Orthopaedic Journal. 1999;12:45-51. Available at: https://upoj.org/ wp-content/uploads/v12/v12_11.pdf.

19. Taillibert S, Bazin B, Pierrot-Deseilligny C. Dysgeusia resulting from internal carotid dissection. A limited glossopharyngeal nerve palsy. J Neurol Neurosurg Psychiatry. 1998;64(5):691692.

20. Rosset E, Albertini JN, Magnan PE, Ede B, Thomassin JM, Branchereau A. Surgical treatment of extracranial internal carotid artery aneurysms. J Vasc Surg. 2000;31(4):713-723.

21. Harriman DG. Ischaemia of peripheral nerve and muscle. J Clin Pathol. 1977;s3-11(1):94-104.

22. Velázquez JM, Montero RG, Garrido JA, Tejerina AA. [Lower cranial nerve involvement as the initial manifestation of Lyme borreliosis]. Neurologia. 1999;14(1):36-37. [Article in Spanish].
23. Cogan A, Boyer P, Soubeyrand M, Hamida FB, Vannier JL, Massin P. Cranial nerves neuropraxia after shoulder arthroscopy in beach chair position. Orthop Traumatol Surg Res. 2011;97(3):345-348.

24. King I, Michla Y, Varma S, Aldridge S. Isolated contralateral glossopharyngeal and vagal nerve palsy after arthroscopic rotator cuff surgery: a case report. J Shoulder Elbow Surg. 2013;22(2):e1-e3.

25. Davies AE, Stone SP, Kidd D, MacMahon J. Pharyngeal sensation and gag reflex in healthy subjects. Lancet. 1995;345(8948):487-488.

26. Johnson LN, Stetson SW, Krohel GB, Cipollo CL, Madsen RW. Aspirin use and the prevention of acute ischemic cranial nerve palsy. Am J Ophthalmol. 2000;129(3):367-371.

27. Fang D, Ran X, Chun W, Zhao T, Tong N, Li X. A clinical analysis of 36 cases with diabetic cranial neuropathy [Abstract no. 2136-PO]. Presented at: American Diabetes Association 64th Scientific Sessions; June 4-8, 2004; Orlando, FL. Available at: https://professional.diabetes.org/ abstract/clinical-analysis-36-cases-diabetic-cranialneuropathy. Last accessed: March 17, 2020.

28. Tankova T, Cherninkova S, Koev D. Treatment for diabetic mononeuropathy with alpha-lipoic acid. Int J Clin Pract. 2005;59(6):645-650.

29. Ruiz J, Varona L, Martín-Gómez JI, Pérez-Bas M, Mateos B, Zarranz JJ. [Spontaneous internal carotid artery dissection as a cause of unilateral lower cranial nerve palsies]. Neurologia. 1995;10(9):391-393. [Article in Spanish].

30. Kashiwamura Y, Sano Y, Abe M, et al. Hydrocortisone enhances the function of the blood-nerve barrier through the up-regulation of claudin-5. Neurochem Res. 2011;36(5):849855 .

\section{Author Affiliations}

Monisha K. Savarimuthu, MBBS, DPM* and Anil K. Nair, $M D+$

*Christian Medical College and Hospital, Department of Psychiatry, Bagayam, Vellore, Tamil Nadu INDIA + Chief of Neurology, Quincy Medical Center; and Director, Alzheimer's Disease Center, Quincy, Massachusetts, USA 